\title{
La nature de la firme et la perspective ouverte par une approche post-marshallienne
}

\section{Gioacchino Fazio}

\section{(2) OpenEdition \\ 1 Journals}

Édition électronique

URL : http://journals.openedition.org/ei/801

DOI : 10.4000/ei.801

ISSN : 2553-1891

Éditeur

Association Économie et Institutions

Édition imprimée

Date de publication : 1 juin 2004

Pagination : $51-70$

ISSN : 1775-2329

\section{Référence électronique}

Gioacchino Fazio, «La nature de la firme et la perspective ouverte par une approche post-

marshallienne », Économie et institutions [En ligne], 4 | 2004, mis en ligne le 31 janvier 2013, consulté le 30 avril 2019. URL : http://journals.openedition.org/ei/801 ; DOI : 10.4000/ei.801

Revue Économie et institutions 


\title{
La nature de la firme et la perspective ouverte par une approche post-marshallienne
}

\author{
Gioacchino Fazio ${ }^{1}$
}

\section{Introduction}

La théorie de la firme a longtemps été structurée autour de la question du comportement des marchés; elle a montré en revanche peu d'intérêt pour une analyse du comportement des firmes (Loasby, 1976). Cet intérêt accordé aux marchés et aux transactions obscurcit sur le plan analytique le rôle de la firme et la nature même de la production. En effet, lorsque la firme est évoquée dans ce type d'analyse, elle ne joue pas un rôle actif dans le système productif, puisque son comportement reste exclusivement conditionné par la structure du marché.

Cette représentation de la firme, qui s'éloigne de la réalité, est à l'origine d'un débat sur sa nature. Ce débat "ontologique» porte sur la manière de représenter la firme dans un contexte théorique dans lequel le marché représente simultanément la forme la plus simple et la plus efficace de coordination des agents économiques. Cependant, la tentative d'expliquer le développement et la diversité des formes d'organisation de la production ouvre de nouvelles réflexions sur la nature de la firme qui se traduisent par le passage d'une analyse à caractère ontologique à une analyse de type "phénoménologique ». Dans cette dernière, la question qui se pose est de savoir dans quelle mesure l'organisation de la firme est fonction de la structure et de la dynamique du marché et d'autres variables « institutionnelles » ou, au contraire, jusqu'à quel point cette organisation est le résultat d'une démarche productive orientée par la firme elle-même, liée principalement à l'évolution de ses propres capacités. C'est grâce à cette nouvelle perspective "phénoménologique» que la théorie de la firme s'intéresse de plus près aux comportement des firmes. Malheureusement, le schéma explicatif proposé par les approches théoriques orthodoxes et autrichiennes, qui se limite à explorer les relations entre la firme et le marché dans un cadre de rivalité, ne semble pas répondre de manière satisfaisante à ces questions plus complexes relatives à la nature des différentes formes d'organisation.

1 Université de Palerme, Dipartimento di Scienze Economiche, Aziendali e Finanziarie, viale delle Scienze, 90128 Palermo (Italia), gfazio@economia.unipa.it.

51 Economie et Institutions $-n^{\circ} 4-1^{\text {e }}$ semestre 2004 
C'est dans le cadre de cette question phénoménologique qu'une approche post-marshallienne est susceptible de fournir des éléments d'analyse intéressants pour les recherches qui s'inscrivent dans la théorie des organisations. En particulier, on montrera que l'idée d' «image», parallèlement au concept de "plan », ouvrent des opportunités de réflexion dans le cadre de l'analyse du processus d'organisation de la production. En effet, la notion d'image offre un point de départ pertinent pour analyser les mécanismes d'acquisition et de coordination des informations, mais aussi pour interpréter les processus de changement et de croissance cumulative de la connaissance dans les organisations. Sur cette base, elle ouvre la perspective d'une interprétation possible, non seulement de la structure de la firme, mais aussi de son évolution.

\section{Les aspects ontologiques de la nature de la firme dans l'approche orthodoxe}

Depuis son apparition dans la littérature et dans les débats théoriques, la question de la nature de la firme se présente principalement comme un problème ontologique. L'objectif est de trouver une justification analytique à l'émergence de la firme dans un contexte théorique dans lequel le marché représente simultanément la forme la plus simple et la plus efficace de coordination des agents économiques. L'identification d'un modèle pour la firme, qui soit à la fois cohérent avec le cadre théorique retenu et représentatif de la réalité productive étudiée, présente des difficultés qui proviennent, au moins en partie, de l'heuristique et de la structure logique de la microéconomie traditionnelle. En effet, cette approche concentre son attention sur les problèmes de rareté des ressources et $d^{\prime}$ allocation des richesses et donc sur le mécanisme du marché et de prix et exclut de facto l'étude des systèmes productifs qui sont à l'origine de la création de la richesse. Le marché représente le lieu privilégié de réalisation des échanges entre agents et de résolution efficace des conflits. Par contre, les prix représentent le mécanisme qui, tout en contenant l'ensemble des informations nécessaires à l'échange, vient garantir une mesure correcte de la valeur des biens et donc le bon fonctionnement du marché.

Initialement, comme le souligne McNulty (1984), cet intérêt accordé au marché et aux transactions a eu pour effet d'obscurcir sur le plan analytique le rôle de la firme et la nature même de la production. Tout en étant présente dans cette analyse, la firme ne joue pas un rôle actif dans le système productif, puisque son comportement de price taker reste fortement conditionné par le système. L'hypothèse de "connaissance parfaite » des objectifs et des techniques disponibles, ainsi que le principe de rationalité, 
impliquent que la firme adopte un comportement efficient que l'économiste est en mesure de déterminer ex ante, à travers la prévision des choix et le calcul anticipé des résultats. Par contre, sur le plan méthodologique, la firme sert de support à l'axiomatique du calcul rationnel de la production et cette dernière se présente dès lors comme le résultat d'une relation entre des unités individuelles (le consommateur et le producteur). La firme apparaît donc selon la définition de S.R. Krupp [dans Machlup F. (1967), p. 10] comme un simple " postulat dans un ensemble d'enchaînements logiques ».

La théorie de la firme essaie de sortir de ce contexte riche de prescriptions normatives pour se confronter de plus près avec le monde réel (i.e. « imparfait »). Le problème est alors de savoir comment intégrer, dans une construction théorique préexistante et déjà structurée, une représentation de la firme plus proche de la réalité ou qui du moins ne la contredise pas. La nécessité d'une telle démarche est liée au fait que le modèle conceptuel dominant rencontre, à l'évidence, de grandes difficultés pour interpréter le fonctionnement des systèmes productifs puisqu'il est représentatif d'une réalité industrielle qui n'a sans doute jamais existée.

Le choix d'une démarche consistant à sortir d'un «monde parfait » a bien évidemment un coût élevé. Elle impose non seulement de s'éloigner des hypothèses fondamentales de la théorie traditionnelle, mais aussi de réexaminer, dans une perspective empirique et parfois inductive, les instruments méthodologiques utilisés. Dans ce nouveau contexte analytique, le renoncement aux axiomes de connaissance et de prévision parfaite, de même que l'abandon subséquent de la conception traditionnelle de la rationalité, entrent en conflit sur le plan logique avec l'idée centrale, dans l'heuristique orthodoxe, de la recherche des conditions qui garantissent l'efficience optimale du système économique.

La solution réside alors dans une tentative de renouvellement partielle qui, tout en ouvrant la porte à l'idée de "rationalité limitée et procédurale» de Simon (1959), permette de conserver une heuristique fondée sur la recherche des conditions objectives garantissant une allocation efficace. Bien qu'il rencontre des obstacles sur le plan logique, ce choix comporte un avantage sur le plan analytique. En effet, par contraste avec l'activité transactionnelle du marché, il est possible d'introduire dans la théorie standard une dimension réellement productive - au sens de making par opposition à buying - et donc, au moins dans une certaine mesure, le concept de firme lui-même.

Le « mécanisme de substitution à la marge » retenu par Coase (1937) fourni ainsi une réponse cohérente à la question ontologique de l'émergence de la firme, mais aussi à la question phénoménologique stricto sensu de savoir pourquoi la firme intègre une série de transactions qui relevait initialement du marché (i.e. problème des frontières de la firme). Elle se présente donc comme un mécanisme d'allocation alternatif au marché dans tous les cas où ce dernier est défaillant. En raison de cette dualité qui

53 Economie et Institutions $-\mathrm{n}^{\circ} 4-1^{\mathrm{e}}$ semestre 2004 
l'oppose au marché, la firme acquière dans ce cadre une physionomie précise - et donc émerge - lorsqu'elle «supplante » le marché. Le fait de placer la firme et le marché sur le même plan lui permet d'aborder le problème de la nature de la firme, sans différencier nécessairement le caractère ontologique du caractère phénoménologique puisqu'il apporte la même réponse aux questions de l'émergence de la firme et de ses frontières. Ce qui explique son succès, bien que tardif, ainsi que l'attachement que la théorie de la firme montre encore à son égard puisqu'elle sert aujourd'hui de soubassement à l'approche transactionnelle, qui se développe à partir de la contribution de Arrow (1969) et Williamson (1972).

Dans ce contexte, les questions de l'organisation et du fonctionnement de la firme ne demandent pas d'autres approfondissements sur le plan analytique. En définitive, peu importe que le modèle théorique du système productif et en particulier de la firme soit peu vraisemblable. En effet, la notion théorique de firme n'est pas construite à l'image de la réalité, mais de manière purement abstraite pour constituer un point de référence idéale permettant d'établir à quelles conditions il est possible d'améliorer ou de « perfectionner » le fonctionnement du marché.

Dans cette optique, bien que la firme "observée » échappe à sa représentation théorique, elle peut malgré tout être "analysée», en tant qu'anomalie résultant des imperfections du marché.

\section{La nature de la firme dans les approches de "style » autrichien: émergence et pérennité}

L'approche orthodoxe de la nature de la firme soulève un problème sur le plan logique, celui de l'absence de toute différenciation dans les critères pour expliquer de l'émergence de la firme (question ontologique) et de sa pérennité, i.e. sa survie et son développement dans le temps (question phénoménologique lato sensu). En effet, pour la théorie orthodoxe, le problème de la pérennité de la firme sur le marché trouve une solution implicite et seulement ex post dans le choix de maximisation de la fonction de profit. Comme l'indique Alchian (1950), ce sont les firmes qui adoptent ce principe qui assure leur pérennité.

Cependant, comme le souligne Langlois (1995), si par leur nature les deux questions (émergence et pérennité) ne peuvent pas être dissociées, elles demandent un approfondissement séparé sur le plan analytique: «(...) la question de l'origine (...) est tout à fait distincte de celle de son fonctionnement, c'est-à-dire, de la manière dont un système de règles une fois établit, se maintient et évolue dans le temps » [Langlois R.N. (1995), p. 253]. Cette distinction se justifie par le fait que le problème de la nature de 
la firme ne peut pas être considéré comme statique parce que la production présente une dimension temporelle qui joue un rôle crucial dans l'organisation de la firme. La raison d'être de cette différenciation réside également dans l'heuristique de l'approche autrichienne. Cette dernière cherche à construire une théorie de la firme à partir de l'intégration de la théorie de l'entrepreneurship [Kirzner I. (1973)] dans une théorie des institutions sociales. La première est la clé d'accès à l'explication de l'émergence de la firme, alors que la seconde permet d'introduire des éléments utiles pour la compréhension des problèmes de fonctionnement ou de pérennité de la firme et donc de certains aspects relevant de la dynamique des organisations. En particulier, la solution au problème de l'existence de la firme paraît évidente dans la mesure où il est possible d'expliquer la naissance de la firme comme le résultat de la capacité de l'entrepreneur à déceler et à saisir des opportunités de profit. C'est cette capacité de discernement qui pousse l'entrepreneur à mobiliser ses efforts pour réunir et coordonner les ressources qui sont nécessaires à sa réalisation. Dans le processus concurrentiel, l'entrepreneur décrit par Kirzner (1992) obtient un profit suite à son action de transformation de la connaissance subjective en connaissance objective. Cette action est essentiellement, sinon entièrement, subjective et elle est, dans une certaine mesure, indépendante des technologies disponibles ou existantes. En effet, dans un contexte de marché caractérisé par des conditions d'incertitude radicale et d'ignorance, l'action de l'entrepreneur est conditionné par les variables considérées comme pertinentes et les possibilités d'action perçues.

En particulier, suite à sa création par l'entrepreneur, la firme se trouve face à une alternative : soit elle peut adopter un comportement traditionnel d'optimisation, soit elle peut développer sa capacité de coordination afin de rendre compatibles les comportements des membres de la firme, guidés par la recherche de gains ou de quasi-rentes. Cette caractéristique de l'action de l'entrepreneur comporte la reconnaissance implicite d'une idée d'opportunisme qui n'est pas très éloignée, mis à part le caractère tacite de la connaissance, de celle proposée dans l'approche néoinstitutionnaliste [Ioannides S. (1989].

Sous cette condition, l'action de l'entrepreneur assure le succès et donc la stabilité de la firme, il peut donc être considéré comme l'élément le plus important qui permet d'en qualifier la nature. Il reste enfin la question de savoir quelle est la nature de cette action de coordination qui permet de définir la firme. En effet, le seul exercice du pouvoir ne suffit pas à caractériser l'activité de la firme parce qu'il se concilie mal avec la liberté que nécessite le processus de découverte. Cette activité va alors être insérée dans une organisation structurée autour d'un système rule-setting, équivalent à celui décrit par Hayek. Dans ce cadre, le problème le plus important est d'identifier le sujet qui détermine les règles, car celles-ci conditionnent la structure dans laquelle agissent les autres agents. Inévitablement, lorsqu'il

55 Economie et Institutions $-\mathrm{n}^{\circ} 4-1^{\mathrm{e}}$ semestre 2004 
s'agit d'un problème d'exercice de pouvoir, dans le cadre des firmes capitalistes, la question est de savoir qui détient le capital. Dès lors, quand on entre dans cette problématique, cette analyse cède le pas à l'approche contractualiste [Hart O. (1995)] qui offre des suggestions plus intéressantes. Le problème des frontières de la firme reste donc un problème qui n'est pas spécifiquement résolu sur la base d'éléments qui relèvent de cette approche de « style » autrichienne.

Cependant, la firme n'apparaît pas comme le moyen de « compenser » les lacunes du marché et donc de rivaliser avec lui, mais elle peut être considérée, dans une certaine mesure, comme le produit du processus de marché. Le modus operandi de la firme ne diffère pas dans son ensemble de celui du marché : comme le marché, la firme promeut l'efficience du système économique à travers la coordination intertemporelle des plans de production et de consommation².

En définitive, le problème des frontières de la firme et de son organisation interne ne peut pas être résolu sur la base d'un calcul à la marge des coûts de transaction, mais il doit être plutôt interprété comme une tentative de structurer et de rendre opérationnel dans la firme les opportunités issues de l'entrepreneurship. De même, la propension de la firme à changer dans le temps peut être elle aussi expliquée par un processus endogène qui est profondément lié au fait que la distribution de la connaissance soit constamment remaniée. Le problème est que cette approche, fondée sur le caractère subjectif du comportement de l'entrepreneur, n'est pas en mesure d'expliquer la pérennité de la firme. Il s'agit en effet d'un problème à caractère dynamique, mais qui dans le cadre de l'analyse autrichienne peut seulement être analysée en termes de statique comparative entre les coûts du marché et ceux de la firme.

Langlois et Robertson (1995) proposent une interprétation différente de la question de la nature de la firme, par rapport aux questions de son émergence et de sa pérennité. En particulier, dans un contexte où les déterminants principaux de l'analyse des formes d'organisation sont la nature de l'incertitude et la structure des compétences ("capabilities»), la firme «émerge» lorsqu'il devient indispensable de coordonner une séquence de stades différents de la production, comme dans les situations d'innovation systémique [Teece, D.J. (1986)].

Comme le met en évidence Langlois (1995), la nécessité de coordonner et de réorganiser en permanence les différentes compétences, en fonction des opportunités de production, oblige à structurer le processus de

2 Cette idée se retrouve chez Rothbard (1994) et Salerno (1993) qui conçoivent le processus de marché comme un mécanisme d'acquisition de connaissance dans lequel la vigilance (alert) et l'habilité de l'entrepreneur à réemployer les ressources ont un rôle fondamental.

Economie et Institutions $-n^{\circ} 4-1^{e}$ semestre 2004 
production en plans. Cette activité de "planning ${ }^{3}$ est quelque chose de différente d'un processus rationnel lié au calcul et à la prévision. En effet, dans un contexte $d$ 'incertitude structurelle et de changement économique radical, le plan de la firme est également le résultat de l'intuition et, surtout, de la vision de l'entrepreneur qui par nature est entièrement subjective. Dès lors, le "planning " ne garantie pas une prévision exacte du cours à venir des actions. Ceci est d'autant plus vrai que ni l'expérience, ni les routines ne peuvent assurer que les résultats de la production seront toujours en conformité avec les anticipations de la firme. Une telle correspondance ne peut être vérifiée qu'ex post.

L'explication de l'émergence de la firme est donc plus liée à un ensemble de conjectures cohérentes qu'à un problème de "planification » dans le sens traditionnel du terme. On comprend alors que dans un système économique en évolution permanente, la raison de l'existence de la firme ne soit pas à rechercher exclusivement dans sa capacité à planifier par un calcul rationnel (ex ante), mais plutôt dans sa vocation à organiser et à réorganiser en permanence (ex post) les capacités - et donc ses règles de conduite - dans le système économique. En définitive, selon Langlois (1984), la firme est la seule organisation capable d'élaborer et d'implémenter de manière efficiente des plans de production, c'est-à-dire à des coûts inférieurs à ceux générés par des mécanismes de coordination décentralisée des activités (i.e. marché). Sur le plan opérationnel, l'avantage comparatif de la firme réside surtout dans la possibilité qu'elle a de dépasser les coûts de transaction «dynamiques » [Nooteboom B. (1992)] liés principalement au changement économique.

Comme on l'a montré, le problème de la nature de la firme atteint une dimension différente quand il s'agit d'expliquer la pérennité. Dans ce cas, ce que la firme devient, dépend surtout de ses capacités à appréhender les sous-systèmes qui la composent et l'environnement dans lequel elle agit. En particulier, en ce qui concerne la question à caractère phénoménologique des frontières de la firme, Langlois (1995) propose à nouveau la dichotomie, introduite par Coase (1937) entre le marché et la firme, mais il montre qu'il est possible de l'interpréter à travers la synthèse des critères utilisés par Hayek (1967) et par Menger (1963) dans l'identification d'une typologie des systèmes de « règles de conduite».

3 Langlois suit la position de Hayek (1967) selon laquelle, chaque système détermine un modèle de guide pour l'action (" preforms on a model the actions it takes"), c'est-à-dire " sets aside a part of itself with which to create a model of the future ". Evidemment, la définition de ce modèle d'action comporte une " céphalisation " de la fonction de décision et de contrôle, que seule la firme est apte à exprimer.

57 Economie et Institutions $-\mathrm{n}^{\circ} 4-1^{\mathrm{e}}$ semestre 2004 
De manière générale, Hayek (1967) propose de classer les institutions à partir de la nature des règles de conduite 4 qui en définissent le comportement. En particulier, selon son expression, il est possible de différencier le système de règles selon qu'elles rentrent dans le cadre du cosmos ("spontaneous market order») ou du taxis ("non spontaneous organizational order $»)^{5}$. Langlois (1993) synthétise ces deux critères de classement des "systèmes de règles " et présente un schéma logique permettant de définir les institutions sur la base de deux paramètres : le processus qui guide l'action (présence ou absence de planification) et la présence d'un objectif dans la définition des règles de comportement. En effet, selon Langlois, la firme naît comme une organisation pragmatique qui poursuit un ou plusieurs objectifs définis par son groupe fondateur ; ces objectifs impliquent l'élaboration d'un programme. Par ailleurs, il est raisonnable de supposer, qu'une fois atteint un certain niveau de réussite, la firme cherche à se pérenniser. Dans cette perspective, le recours au marché est plus efficace parce qu'il se présente comme un meilleur système d'incitations. En effet, à long terme, les routines et les institutions sociales permettent d'atténuer l'opportunisme et de réduire ainsi les coûts de transaction. Une fois l'innovation assimilée et diffusée à travers la production de masse, le processus d'internalisation devient plus coûteux pour la firme qui recourt alors à l'externalisation. De ce point de vue, cette approche de «style » autrichien rejoint aussi l'approche évolutionniste dans la mesure où, comme Langlois l'admet, la meilleure manière pour la firme de planifier un avenir difficile à prévoir est de simuler un ordre spontané. La firme reproduit alors le comportement du marché.

En définitive, bien qu'il soit possible d'établir une distinction entre la firme et le marché - reposant sur l'idée que la première est une organisation finalisée poursuivant un objectif, tandis que le second est une institution abstraite - cette distinction est plus liée au déroulement du processus d'apprentissage qu'à la nature de la règle de conduite qui caractérise ces deux formes d'organisation. Ce processus d'apprentissage, par lequel la

4 Selon Dworkin (1977), il faut faire une distinction entre les principes et les règles. Les premiers comprendraient les valeurs, les objectifs et l'ensemble des préférences; les secondes sont finalisées à l'obtention de l'efficience. Dans ce cas, le sens dans lequel Hayek utilise le terme de "règles ", doit être interprété de manière large, qui prend en considération les principes comme les règles.

5 Alors que le cosmos est constitué d'un ensemble de règles abstraites qui ne poursuivent pas un objectif particulier, le taxis comprend des règles de comportement qui engagent ses membres à la réalisation d'un objectif, plus ou moins concret. De manière différente, Menger (1963) introduit la distinction entre les systèmes pragmatiques, dont le comportement est fonction d'un processus de "planning ", et les systèmes organiques, dont le devenir est le résultat d'un processus non intentionnel. 
firme peut manifester son avantage comparatif par rapport au marché, n'est pertinent que dans un contexte d'innovation systémique; en dehors de ce contexte la firme n'est pas en mesure de supplanter le marché.

La vision qui émerge, non seulement n'est pas contraire à la conception dichotomique de la firme et du marché, mais elle contribue même à la renforcer. Néanmoins, un élément de distinction serait lié au fait que, alors que dans l'approche de «style» autrichien l'élément du "pragmatisme» explique seulement l'émergence de la firme, dans l'approche orthodoxe il permet aussi d'expliquer ses frontières.

En outre, à la différence des approches mainstream, dans l'approche de «style » autrichien, les règles de conduite qui définissent la nature de la firme impliquent non seulement des conditionnements, mais présupposent aussi des « habitudes ${ }^{6}$. Cette conception, selon Langlois, a été approfondie et rendue opérationnelle par l'approche évolutionniste, à travers l'idée de routines, mais aussi par les approche basée sur les compétences ${ }^{7}$, qui analysent les effets de l'application de ces règles de comportement sur les différents types d'organisation. En effet, dans ce cadre, les règles de conduite, de même que les routines que les individus suivent dans l'organisation, rendent compte de la connaissance, souvent tacite, indispensable à leur action. Cette connaissance se traduit dans la firme par un ensemble de «compétences productives ». Dès lors, la quintessence de l'organisation de la firme ne réside pas dans l'analyse de ses transactions, mais dans l'analyse de son activité de production. Dans ce cadre, la question de la "pérennité » de la firme devient alors celle de savoir comment elle acquiert, transmet et protège ses capacités productives.

Cependant, même si cette analyse de la firme en termes des compétences semble accorder à la firme un statut spécifique qui lui permet de s'émanciper d'une analyse fondée sur l'échange, elle rejoint néanmoins l'approche néo-classique sur la question de la nature de la firme. En effet, comme le souligne Langlois (1995), l'essence du marché et de la firme est au fond la même, puisqu'ils coordonnent tous les deux des compétences: « (...) Les organisations et les marchés sont composés d'individus qui savent comment faire certaines choses et qui poursuivent des objectifs différents. La question est de savoir comment - et pourquoi - ces compétences se soudent parfois dans une organisation » (p. 258). Dans ce cadre, pour résoudre le problème de la nature de la firme il suffit de répondre à la question : pourquoi et comment ces compétences se distribuent-elles entre le marché et l'organisation ? A cette question Langlois et Robertson (1995) apportent la

6 Sur ce point, l'analyse de Langlois rejoint l'idée de Simon (1959) qui conçoit plus l'individu comme un agent " programmé " pour suivre des règles de comportement, que comme un agent qui agirait toujours de manière parfaitement consciente.

7 Cf. Teece et al. (1992), Chandler (1992), Langlois (1998), Loasby (1998, 1999).

59 Economie et Institutions $-\mathrm{n}^{\circ} 4-1^{\mathrm{e}}$ semestre 2004 
réponse suivante: «(...) Les frontières de la firme sont déterminées (au moins en partie) par les coûts de développement interne de ses capacités complémentaires ou de leurs acquisition à travers des contrats avec les autres firmes $»($ p. 31).

Comme dans le cadre de l'approche néo-classique, le problème de la définition des frontières entre la firme et le marché est abordé en termes de make-or-buy. La seule différence est que cette fois l'unité d'analyse n'est plus la transaction mais la compétence ou les routines.

\section{La nature de la firme et le "planning" dans l'approche post marshallienne}

Dans l'étude de la nature de la firme, le mainstream se concentre plus sur l'identification de la firme comme "mécanisme» de centralisation du processus d'information qu'à l'approfondissement du rôle central que la firme a dans l'organisation de l'activité productive et, par conséquent, dans l'évolution des systèmes productifs en général. L'école autrichienne, qui revient sur cette question par le biais du concept de "planning ", cherche à établir, selon les circonstances, quel est le « système de règles de conduite »l'organisation (firme) ou l'institution (marché) - qui permet une meilleure «économie de connaissance » face au changement économique.

Bien que cette approche ait été encore peu approfondie par la théorie économique, elle garde une place importante dans la tradition postmarshallienne. En particulier, le concept de «plan» est fondamental parce qu'il permet non seulement de mieux comprendre la nature de la firme, mais aussi la manière dont elle interfère et interagit avec les activités productives des autres firmes et donc avec son environnement (i.e. l'industrie). Dans ce cadre, Edith Penrose remarque que : « (...) la première fonction économique de la firme industrielle est de faire usage de ses ressources productives dans le but d'offrir des biens et des services en concordance avec les plans anticipés et mis en œuvre au sein de la firme " [Penrose E.T. (1995), p. 15]. Par cette affirmation, Penrose ne se limite pas à signaler la fonction principale de la firme, elle souligne également le fait que cette fonction prévoit la définition d'un " plan » de production. En particulier, Penrose, qui adopte implicitement une distinction introduite par Ryle (1949), indique que dans la firme cette activité de programmation se déroule en deux étapes : la première permet la définition d'un plan d'expansion (quoi faire), tandis que la seconde conduit à la réalisation de ce plan à travers des modalités de savoir-faire spécifique («know how»); ce qui posent le problème de la 
production (comment faire) ${ }^{8}$. Bien qu'elles soient souvent analysées séparément, ces deux étapes restent étroitement liées et interdépendantes, parce qu'elles se déroulent de manière séquentielle. En effet, la définition du processus conduisant à l'exécution des plans de production est à l'origine des nouvelles modalités de savoir-faire, qui, à leur tour, se reflètent dans la définition des objectifs futurs de la firme.

Cette différenciation est également acceptée par Loasby (1996) qui établit une distinction entre deux formes de connaissance que sont le «knowing that » et le «knowing how» (i.e. les capacités). Il reconnaît qu'elles peuvent avoir une nature différente puisque «(...) c'est possible d'être un bon exécutant (performer) sans découvrir quels sont les principes qui sont liés à la performance ou, simultanément, d'être compétent dans les principes et un mauvais exécutant » [Loasby B. (1996), p. 90].

En effet, si le processus qui conduit à la définition des objectifs (qui répond à la question quoi faire) est liée à l'évaluation subjective des opportunités productives et des ressources disponibles, ou éventuellement disponibles, le processus de réalisation ou de production (qui répond à la question comment faire) est plus difficile à interpréter et à définir ex ante. Penrose concentre principalement son attention sur l'analyse de ce dernier processus. En particulier, elle commence par considérer le processus de production comme un processus de développement des services et de création de compétences. Elle admet également que l'activité de coordination des services productifs peut être considérée comme le résultat d'un processus décisionnel de type administratif, qui se déroule en fonction de l'expérience accumulée par chaque firme.

Richardson (1998) adhère complètement à cette interprétation qui a pour conséquence de caractériser la firme par sa capacité de coordination et de programmation du processus de production. Contrairement à Coase (1937), Richardson soutient en effet que la firme n'émerge pas fondamentalement parce que le recours au mécanisme des prix devient coûteux, mais parce qu'elle est apte à coordonner le processus productif, c'est-à-dire à exécuter simultanément des actions ("concurrent coordination ») qui sont en conformité avec le plan de production qu'elle

8 Knight (1921) a peut-être été l'un des premiers à suggérer, qu'en présence d'incertitude, les deux problèmes "what " et "how " acquièrent une importance décisive par rapport à la simple "exécution " et à souligner que, en conséquence, le problème de l'organisation interne de la production ne peut pas se réduire à une simple " (...) matter of indifference or a mechanical detail " [Knight F.H. (1921), p. 268]. Pour Knight, dans la vie économique réelle, la centralisation des moments de décision et de contrôle se réalise à travers un processus de " céphalisation " comparable à celui qu'on observe dans l'évolution de la vie organique. Cependant, dans les années qui ont suivi, les suggestions de Knight, critiquées par Coase (1937), n'ont suscité qu'un faible intérêt parmi les théoriciens de la firme.

61 Economie et Institutions $-\mathrm{n}^{\circ} 4-1^{\mathrm{e}}$ semestre 2004 
s'est fixé. Il en résulte que cette activité de coordination ne peut pas être considérée, d'un point de vue analytique, comme un effet direct et non voulu, déterminée par les caractéristiques des transactions de marché. Richardson considère en effet que, sans le «design » et la «direction », la coordination permanente d'une gamme étendue d'activités complémentaires ne serait pas possible.

Cependant, Richardson admet également que les firmes effectuent aussi des transactions et des échanges; elles sont alors indirectement à l'origine d'un ordre spontané constitué par un ensemble de typologies de biens et de quantités produites. Ce mécanisme, qui n'est autre que le marché, influe sur les plans de la firme car il conditionne les sentiers de production et, plus généralement, l'interaction entre des décisions qui se succèdent de manière indépendante. Ainsi il stimule un changement graduel, fait d'ajustements successifs. Cette coordination par le marché est alors séquentielle et évolutive. Il en résulte que le processus de planification est lui-même largement conditionné par les imperfections du marché, mais c'est grâce à ces imperfections que les capacités productives de la firme émergent et deviennent profitables. Il est ainsi possible de constater, en reprenant les termes de Loasby, que : "les économies réelles se stabilisent grâce à de nombreux phénomènes qui représentent des imperfections selon l'idéal walrasien, mais qui sont nécessaires pour le fonctionnement efficace de l'économie » [Loasby B. (1996), p. 93].

Dans ces conditions, les activités productives de la firme peuvent être représentées comme le résultat des anticipations des opportunités productives réalisées par la firme. Le développement de ces opportunités est au cœur des mécanismes de croissance de la firme, comme leur restriction en constitue la limite. De ce point de vue, l'apporte essentiel de Penrose réside dans le fait qu'elle souligne d'une part, que la possibilité de créer de nouvelles compétences et de nouvelles connaissances se situe sur le versant subjectif des déterminants économiques de l'activité de la firme et, d'autre part, que ces connaissances et ces compétences sont à l'origine de l'organisation même de la production.

Cette approche post-marshallienne de la firme rejoint le concept de firme proposé par l'approche béhavioriste de Cyert (1988). Pour cet auteur, même si le recours à la règle marginaliste de détermination des prix permet de mieux comprendre comme les prix opèrent et les aspects les plus significatifs de l'allocation des ressources économiques, elle ne permet pas d'appréhender le caractère spécifique du processus de décision de la chaque firme. En effet, quand on sort d'un cadre économique caractérisé par la concurrence parfaite pour entrer dans un environnement productif incertain, seul le processus de programmation introduit par la firme permet de structurer le processus de production. En revanche, en l'absence d'incertitude, l'activité productive peut être parfaitement structurée et il n'est plus nécessaire pour la firme d'élaborer de plan.

Economie et Institutions $-n^{\circ} 4-1^{e}$ semestre 2004 
D'autre part, puisque dans des conditions d'incertitude la référence à la maximisation du profit ne permet pas d'expliquer l'existence de plans de production différents, la construction d'un critère permettant de guider les actions de la firme ne peut plus se fonder sur un tel principe. C'est pour cette raison que la firme, à travers son équipe managériale, élabore un plan de production qui prévoit une succession d'actions. En particulier, ce plan consiste, selon Cyert (1988), en : «(..) une série de cibles par rapport à certaines variables critiques. Ces cibles sont développées sur une base à la fois mensuelle et annuelle. Ils sont spécifiés pour la firme dans son ensemble aussi bien que pour les divisions. La prise en considération des cibles et des positions initiales de la firme et de ses divisions lui permettent de développer des stratégies spécifiques et tactiques pour atteindre l'objectif fixé » (p. 55). Si ces objectifs choisis par les managers ont une probabilité raisonnable de se réaliser, le processus qui conduit à la définition du plan de production représente, selon Cyert, une sorte de prévision. Dans ce processus de planification, chaque firme est confrontée à une analyse cognitive qui la conduit à formuler des conjectures à partir de trois ensembles de conditions: (1) la conjoncture économique dans son ensemble, (2) la situation de l'industrie et des concurrents, (3) la situation interne de la firme. Dans la construction de son processus décisionnel, la firme doit tout d'abord, à partir d'un examen des deux premières conditions, pouvoir calculer les ventes prévues dans l'industrie, ensuite, après avoir évaluer sa propre situation interne, la firme peut se confronter avec son environnement externe pour essayer de définir le plan de production qui lui paraît le plus plausible pour la période considérée.

\section{Le concept d'image dans l'interprétation post-marshallienne de la nature de la firme}

Dans son ouvrage intitulé The Image (1956), Kenneth Boulding propose une interprétation du processus cognitif et productif dans les organisations. Selon Boulding, l'idée que la connaissance trouve son origine et prend forme dans une série d'images que chaque individu construit de manière différente, par rapport au présent et au futur, et qui représentent à la fois son propre environnement, la position qu'il occupe en son sein, mais aussi ses valeurs et ses objectifs.

Le processus qui conduit à l'élaboration et à la réalisation de ces images peut être considéré comme de fondement de l'ensemble des activités de l'homme et, en particulier, de son activité productive et des ses résultats (human artefacts). Boulding inclut dans ces résultats les organisations économiques. Ce processus appartient seulement à l'homme et ne peut pas

63 Economie et Institutions $-\mathrm{n}^{\circ} 4-1^{\mathrm{e}}$ semestre 2004 
être étendu au reste du genre animal, dont le comportement et l'évolution peuvent être plus correctement interprété par les lois de la biogénétique et par le processus de sélection naturelle. Selon Boulding, la place que prend ce processus de sélection dans la sphère non humaine est occupée, dans la sphère humaine, par la capacité d'imaginer et, à travers elle, par la capacité de faire. Cette position n'est pas isolée dans le courant post-marshallien. Loasby (1999) souligne également que l'analyse de l'évolution de la société humaine et celle des autres espèces animales ne sont pas assimilables parce qu'elles ne se fondent pas sur les mêmes principes. L'action des êtres humains est, en effet, « résolue » intentionnelle ; elle est le résultat d'un plan. De même la diminution ou l'augmentation de la variété dans le système économique et donc l'innovation sont souvent le résultat d'un "dessein ", d'un projet de l'homme. Or, ces aspects ne trouvent pas d'équivalent dans le modèle biologique.

Malheureusement, l'analyse de Kenneth Boulding se limite seulement à prendre en compte la relation entre l'élaboration des différentes images et le comportement de l'individu et des organisations. Le degré de correspondance de l'image en tant que représentation de l'esprit à la réalité, et, par la suite, l'évolution de cette représentation dans le temps, n'intéresse pas Boulding qui semble éluder cette question. Ce problème peut cependant fournir de nouveaux éléments d'interprétation concernant la dynamique des organisations productives.

C'est dans cette perspective que s'oriente l'analyse du concept d'image proposée par Edith Penrose dans son ouvrage The Theory of the Growth of the Firm (1995). A ce propos, il nous semble opportun d'observer que Penrose semble vouloir revendiquer pour elle-même les mérites de l'introduction de cette idée : « (...) Ces lignes étaient écrites dans une forme légèrement différente avant l'apparition du petit ouvrage imaginatif écrit par Kenneth Boulding » (p.5). En effet, cette mise au point nous montre que Penrose considère que le concept d'image joue un rôle important dans son approche théorique et que, plus généralement, elle lui attribue un grand potentiel dans l'étude de la firme. Cependant, la référence à l'idée d'image, bien qu'essentielle à l'analyse penrosienne, est très brève. Elle se limite à la partie introductive de son ouvrage, dans laquelle elle explique les origines et la nature de la firme en tant qu'organisation ${ }^{9}$.

Dans ce cadre, Penrose critique la justification avancée par la théorie néo-classique pour expliquer l'émergence de la firme. En particulier, elle observe que, même si la récolte efficace d'informations sur les événements futurs peut expliquer sur le plan analytique la naissance de la firme, cette dernière se caractérise plus par la manière dont les sujets qui la composent interagissent avec les informations recueillies, que par sa capacité à récolter

9 Cette partie a été en particulier réexaminée par Penrose en 1995, dans la troisième édition de son ouvrage.

Economie et Institutions $-n^{\circ} 4-1^{e}$ semestre 2004 
de l'information. En effet, toute l'organisation de l'activité productive se fonde sur la perception que la firme a de la pertinence de ses anticipations, plus que sur une évaluation objective de cette pertinence.

Penrose propose alors d'analyser les choix d'organisation et de production de la firme à partir de l'étude des facteurs qui déterminent l'action de l'entrepreneur. Ce changement de perspective implique la réouverture de la question du statut de l'entrepreneur. Selon Penrose, en effet, les capacités managériales (fondées sur les compétences et sur les expériences) et le "tempérament» de l'entrepreneur sont les facteurs qui permettent de mieux évaluer les choix de la firme, dans le cadre d'un environnement caractérisé par l'incertitude. Or, ces facteurs qui sont à l'origine du comportement subjectif des firmes ne peuvent pas être expliqués en dehors du système d'où ils émergent. Dans le cadre de la théorie de la firme, la structure de ce système qui correspond au marché est alors indispensable pour interpréter le comportement de l'organisation et elle doit être considérée selon Penrose : «(...) comme étant, dans la conscience des entrepreneurs, l'image des possibilités et des limites qu'ils rencontrent : c'est finalement cette «image» qui détermine en fait le comportement d'un individu » [Penrose E.T. (1995), p. 5].

Edith Penrose ajoute un élément nouveau par rapport au raisonnement introduit par Boulding sur les «human artefacts » : c'est la prise en compte de l'environnement auquel généralement chaque individu fait référence lorsqu'il définit son propre programme d'action. L'acceptation de cette démarche logique et sans doute peu originale a néanmoins des implications intéressantes sur le plan théorique.

Avant tout, la logique explicative qui permet d'identifier un critère pour tracer la frontière optimale entre les transactions réalisées à l'intérieur et les transactions réalisées à l'extérieur de la firme (the boundaries of firms) devient plus complexe par rapport à celle qui émerge suite aux débats sur la nature de la firme et sur sa taille optimale. En effet, le marché lui-même n'est pas considéré comme objectivement extérieur à la firme, mais il est inscrit dans un espace mentale élaborée par l'entrepreneur. C'est à partir de cette espace mental que ce dernier exploite ses propres opportunités productives et sur la base duquel il interagit avec le système productif dans son ensemble. Ainsi, il n'est pas possible en pratique de définir à priori les frontières de cette espace, comme dans le cas des objets inanimés, c'est-àdire en faisant référence à l'espace occupée. En effet, l'environnement interne et l'environnement externe à la firme sont composés d'une série $\mathrm{d}^{\prime}$ ' «images créées » par l'entrepreneur sur la base d'un amalgame de faits et de valeurs, considérés comme vrais ou possibles, mais de toute façon vérifiables et par la suite susceptibles de modifications dans le temps ${ }^{10}$.

10 L'étude de ce processus cognitif mené par des sujets "actifs " par l'interaction entre construction et validation (qui sont des cognitive acts) fait

65 Economie et Institutions $-\mathrm{n}^{\circ} 4-1^{\mathrm{e}}$ semestre 2004 
De plus, le processus qui conduit à la création d'images précède dans l'ordre logique le processus d'acquisition des informations, de coordination et de contrôle des moyens de production et parfois est plus important. Pour cette raison, une approche fondée uniquement sur une théorie de l'information fournit une vision incomplète et restrictive de l'origine et la nature de la firme. En somme, bien que l'identification et la réalisation d'un processus productif, (i.e. processus créateur) soit influencée par la structure de l'information, celle-ci passe avant tout par l'élaboration de l'image à l'intérieur d'un modèle qui prévoit et pourvoit à l'action [Glasersfeld E. (1981)], en projetant une série d'actions et réactions (i.e. des stratégies). En effet, par la définition de ses propres plans d'action, la firme ne se limite pas à une évaluation simple du contexte productif, mais imagine comment pouvoir le modifier afin de réaliser ses objectifs. Dans ce processus, les connaissances accumulées, l'expérience acquise et la capacité d'interpréter interagissent de différentes façons. Le résultat de ce processus, l'image, est à l'origine de la manière différente dont chaque firme perçoit les «conditions de la demande » et vient donc expliquer la différence observée entre la dynamique de la firme et celle de l'industrie (i.e. l'unicité de chaque sentier de croissance).

Les ajustements dans le temps de l'image conçue et les changements dans le processus d'imagination ne sont pas uniquement conditionnés par le passé de la firme. En effet, s'il est vrai que la dynamique du sentier évolutif de la firme est déterminée, dans une large mesure, par les connaissances acquises au cours du temps et, conjointement, par l'évolution des ses ressources, cependant, il n'est pas toujours vrai que le franchissement des limites connues est un processus qui peut être interprété dans le cadre d'une analyse des processus cognitifs liés à l'acquisition d'informations. D'autre part, comme la goals approach [Simon H.A. (1991)] le met en évidence, tout en privilégiant une analyse du comportement en termes de «causes efficientes", on finit par négliger les "causes finales", essentiels dans l'étude des organisations productives. Cet aspect, sous-estimé dans l'approche évolutionniste, peut être récupéré si on réussît à rendre l'idée d'image plus opérationnelle.

Cependant, les économistes post-marshalliens qui se sont intéressés à la notion d'image ne semblent pas emprunter cette orientation. L'analyse de ce thème a subi un arrêt prévisible, lié au fait cette question relève plus aujourd'hui des sciences de gestion. Plus récemment pourtant, c'est dans cette direction que semble s'orienter les recherche de Martin Fransman (1994, 1998). Le renouveau de l'intérêt pour cette question se traduit par un dépassement de l'idée que l'image serait le seul produit de l'interprétation de la firme par rapport à sa position concurrentielle, comme semblent le

l'objet d'un approfondissement dans l'analyse construtiviste [Watzlawick P. (1981), Tamborini R. (1997)].

Economie et Institutions $-n^{\circ} 4-1^{e}$ semestre 2004 
faire les études à caractère managériale. En effet, les conditions du marché et le critère du choix efficient des opportunités productives courantes (i.e. l'expansion d'inputs déjà existants) de la part de la firme permettent $\mathrm{d}$ 'expliquer seulement une partie du phénomène de la croissance de la firme. Cette vision restrictive de l'acception du concept d'image ne permet pas de clarifier la dynamique des comportements de l'organisation et réduit, encore une fois, l'essence de l'activité productive à un problème de coordination. En outre, le comportement de la firme est le plus souvent déterminé par la manière dont la firme imagine d'utiliser ses ressources productives présentes et éventuellement disponibles. Dans ce cas, les opportunités productives dépendent de ce que la firme est en mesure de réaliser. A ce propos, Day décrit ainsi cet aspect de l'activité de la firme: "Les inventeurs et les entrepreneurs imaginent des choses qui n'existent pas et prévoient des étapes pour la réalisation, tout comme chacun de nous fais lorsqu'il formule un plan pour le futur et le réalise » [Day R.H. (1995), p. 1477].

Or, ce que la firme imagine pouvoir produire ne se fonde pas seulement sur un degré de connaissance objective et de nature atemporelle du marché, des préférences et des prix, mais est plutôt lié au processus de recherche par lequel la firme identifie et pense augmenter ses propres ressources productives. Alors, les mécanismes qui déterminent l'évolution de l'organisation de la firme ne dépendent pas seulement de la gestion ou de la « révélation» des informations sur les opportunités d'investissement et de croissance, mais ils dépendent tout autant de l'interprétation subjective des opportunités possibles et de la croissance cumulative de la connaissance collective $^{11}$. Ces opportunités ne proviennent pas de la dimension objective de l'activité de la firme, mais de sa dimension subjective. En effet, de telles opportunités productives peuvent changer ou apparaître en absence de toute variation des conditions externes et des techniques de production; il suffit d'un simple changement de la manière dont l'entrepreneur imagine son activité et le marché auquel il s'intéresse. Il en résulte donc que ces opportunités productives n'ont aucune raison d'être présentes ou prévisibles au moment de la réalisation du programme d'expansion de la firme.

Finalement, dans l'analyse post-marshallienne la firme ne peut pas se réduire à être un lieu d'accumulation et de coordination de compétences productives spécifiques (i.e. repository of productive knowledge). Elle est également un centre d'observation et d'imagination du système dans lequel s'inscrit son action et avec lequel elle interagit. Ainsi, cette firme ne peut pas non plus être considérée comme agent passif, soumis au jeu perpétuel de l'innovation, comme c'est le cas dans l'approche néo-classique et aussi en

11 Cette idée est pour Normann (1977) à la base de la construction d'une formule clé de succès. Cette formule, qu'il appelle "business idea ", est en effet le résultat d'un processus d'apprentissage entrepreneurial qui commence avec la "vision d'anticipation " de la firme.

67 Economie et Institutions $-\mathrm{n}^{\circ} 4-1^{\mathrm{e}}$ semestre 2004 
partie dans l'approche évolutionniste. Elle est au contraire l'agent dont l'action est à l'origine de cette innovation. En d'autres termes, La firme postmarshallienne n'est pas le vecteur assurant la réalisation de l'innovation dans le cadre d'une logique d'efficience, mais bien le moteur de la croissance économique.

\section{Bibliographie}

Alchian, A.A. [1950], "Uncertainty, Evolution and Economic Theory », Journal of Political Economy, vol. 68, n 58, p. 211-221.

Arrow, K.J. [1969], « The Organization of Economic Activity: Issues Pertinent to the Choice of Market versus Nonmarket Allocation » in The Analysis and Evaluation of Public Expenditure: The PPE System, U.S. Government Printing Office, Washington.

Boulding, K.E. [1956], The Image, University of Michigan Press, Ann Arbor. Chandler, A.D. [1992], «Organizational Capabilities and the Economic History of the Industrial Enterprise », Journal of Economic Perspetives, vol. 6, $\mathrm{n}^{\circ}$ 3, Summer, p. 79-100.

Coase, R.H. [1937], « The Nature of the Firm », Economica, vol. 4, n.s., Spring, p. 3-47.

Cyert, R.M. [1988], «Towards a Control Theory of the Firm » in The Economic Theory of Organization and Firm, Harvester Wheatsheaf.

Day, R.H. [1995], «Rationality, Entrepreneurship and Institutional Evolution ", Revue Economique, n 6, p. 1473-1485.

Dworkin, R.M. [1977], Taking Right Seriously, Duckworth, London.

Fransman, M. [1994], «Information, Knowledge, Vision and Theories of the Firm », Industrial and Corporate Change, vol. 3, n 3, p. 713-755.

Fransman, M. [1998], « Analysing the Evolution of Industry: The Relevance of the Telecommunications Industry», Competences, Governance and Entrepreneurship, Summer Conference, Bornholm, 9-11 Juin.

Glasersfeld, E. [1981], «Introduction to Radical Constructivism» in P. Watzlawick (ed.) [1981], The Invented Reality, Norton, New York.

Hart, O. [1995], Firms, Contracts and Financial Structure, Clarendon Press, Oxford.

Hayek F.A. von [1967], Studies in Philosophy, Politics, and Economics, University of Chicago Press, Chicago.

Kirzner, I. [1973], Competition and Entrepreneurship, University of Chicago Press, Chicago.

Kirzner, I. [1992], «Entrepreneurship, Uncertainty and Austrian Economics ", in B.J. Caldwell et S. Boehm (eds.), Austrian Economics: Tensions and New Directions, Kluwer Academic Publisher, Boston. 
Knight, F.H. [1921], Risk, Uncertainty, and Profit, Boston: Houghton Mifflin. University of Chicago Press, Réimprimé 1971, Chicago.

Ioannides, S. [1998], "Towards an Austrian Perspective on the Firm », Review of Austrian Economics, vol. 11, $\mathrm{n}^{\circ} 2$.

Langlois, R.N. [1984], «Internal Organization in a Dynamic Context: Some Theoretical Considerations", in M. Jussawalla et H. Ebenfield (eds.), Communication and Information Economics: New Perspectives, North-Holland, Amsterdam, p. 23-49.

Langlois, R.N. [1993], «Orders and Organizations: Toward an Austrian Theory of Social Institutions », in B. Caldwell et S. Böhm (eds.), Austrian Economics: Tensions and Directions, Kluwer Academic Publishers, Dordrecht. Langlois, R.N. [1995], «Do Firms Plan?», Costitutional Political Economy, vol. $6, \mathrm{n}^{\circ} 3$, p. $247-261$.

Langlois, R.N. et P.L. Robertson [1995], Firms, Markets and Economic Change. A Diagnostic Theory of Business Institutions, Routledge, London and New York.

Langlois, R.N. [1998], "Capabilities and Theory of the Firm », in N.J. Foss et B.J. Loasby (eds.), Economic Organization, Capabilities and Co-ordination, Essays in Honour of G.B. Richardson, Routledge, London and NewYork, p. 183-203.

Loasby B.J. [1976], Choice, Complexity and Ignorance, Cambridge University Press, Cambridge.

Loasby, B.J. [1996], "The Organisation of Industry and the Growth of Knowledge ", Vortragsreihe, 'Lectiones Jenenses', Schriftenreihe des M.P.I. zur Erforschung von Wirtschaftssystem, Heft 7, Bad Blankenburg: Harfe-Verlag und Druckerei GmbH.

Loasby, B.J. [1998], «The Organisation of Capabilities », Journal of Economic Behavior \& Organization, vol. 35, p. 139-160.

Loasby, B.J. [1999], "Cognition, Capabilities and Co-operation", Comportements Coopératifs, Ecole de Sophia Antipolis sur la Firme et l'Industrie, 22-27 November.

Machlup, F. [1967], «Theory of the Firm: Marginalist, Behavioural, Managerial », American Economic Review, vol. 57 (1), p. 1-33, in A.M. Cardani et U. Pedol (eds.) [1980], Problemi di Teoria dell'Impresa, Etas Libri, p. 135-174.

McNulty, P. [1984], « On the Nature and Theory of Economic Organization: the Role of the Firm Reconsidered », History of Political Economy, vol. 16, $\mathrm{n}^{\circ} 2$.

Menger, C. (1963) [1883], Problems of Economics and Sociology, Trans. F.J.Nock, University of Illinois Press, Urbana.

Nooteboom, B. [1992], "Towards a Dynamic Theory of Transactions », Evolutionary Economics, $\mathrm{n}^{\circ}$ 2, p. 281-299.

Normann, R. [1977], Management for Growth, John Wiley \& Sons Ltd, Chichester.

Penrose, E.T. [1995] (1959), The Theory of the Growth of the Firm, Oxford University Press, 3 édition, Oxford.

69 Economie et Institutions $-\mathrm{n}^{\circ} 4-1^{\mathrm{e}}$ semestre 2004 
Richardson, G.B. [1998], "Some Principles of Economic Organisation », in N.J. Foss et B.J. Loasby (eds.), Economic Organization, Capabilities and Coordination, Essays in Honour of G.B. Richardson, Routledge, London and NewYork.

Rothbard, M. [1994], «Book Review (of Austrian Economics: Tensions and New Directions) ", Southern Economic Journal, October.

Ryle, G. [1949], The Concept of Mind, Hutchinson, London.

Salerno, J. [1993], " Mises and Hayek Dehomogenized », Review of Austrian Economics, vol. 6, $\mathrm{n}^{\circ} 2$.

Simon, H.A. [1959], "Theories of Decision Making in Economics and Behavioral Science », American Economic Review, vol. 49, June, p. 253-283.

Tamborini, R. [1997], «Knowledge and Economic Behaviour. A Constructivist Approach», Journal of Evolutionary Economics, vol. 7, $\mathrm{n}^{\circ} 1$, p. 49-72.

Teece, D.J., G. Pisano, A. Shuen et P.L. Robertson [1992], «Dynamic Capabilities and Strategic Management», Working Papers, University of California, Berkeley.

Watzlawick, P. (ed.) [1981], The Invented Reality, Norton New York.

Williamson, O.E. [1972], "Antitrust Enforcement and the Modern Corporation », in V.R. Fucks [1972], Policy Issues and Research Opportunities in Industrial Organization, N.B.E.R. and Columbia University Press. 